\title{
La communication interne : une approche croisée
}

Béatrice Galinon-Mélénec

\section{OpenEdition}

Journals

Édition électronique

URL : http://journals.openedition.org/communicationorganisation/1695

DOI : 10.4000/communicationorganisation. 1695

ISSN : 1775-3546

\section{Éditeur}

Presses universitaires de Bordeaux

\section{Édition imprimée}

Date de publication : 1 mai 1994

ISSN : 1168-5549

\section{Référence électronique}

Béatrice Galinon-Mélénec, «La communication interne : une approche croisée », Communication et organisation [En ligne], 5 | 1994, mis en ligne le 26 mars 2012, consulté le 01 mai 2019. URL : http:// journals.openedition.org/communicationorganisation/1695; DOI : 10.4000/ communicationorganisation. 1695

Ce document a été généré automatiquement le 1 mai 2019.

(c) Presses universitaires de Bordeaux 


\title{
La communication interne : une approche croisée ${ }^{1}$
}

\author{
Béatrice Galinon-Mélénec
}

1 En 1993, le Comité National d'Évaluation² recommandait à la recherche en information et communication de «développer des espaces (revues, publications diverses) » dans le domaine (...)des $\mathrm{SIC}^{3}$ aux enseignants-chercheurs de "s'investir dans la recherche appliquée en faisant mieux connaître leurs activités et en s'informant des besoins des entreprises »(...), d'ouvrir des voies de collaboration avec les écoles de commerce et de gestion ${ }^{4}$ dont «les enseignants permanents ont des activités de recherche et de publication en ce domaine, (certains exprimant) le souhait d'établir des liens avec des enseignants-chercheurs universitaires dans une optique de recherche fondamentale ».

Le dossier consacré à la communication interne des organisations est construit dans une tentative de réponse à cette demande. On y trouvera non seulement des articles d'universitaires français et étrangers mais aussi des articles de chercheurs des écoles supérieures de gestion. La diversité de l'approche vise à repérer les différents points d'entrée du champ que constitue la communication interne. Elle est complétée, hors dossier, d'articles de professionnels de la communication.

3 Sur cette trame, à la fois théorique et pratique, peut se développer une réflexion transdisciplinaire dont l'objet est le champ managérial.

4 Les auteurs soulignent que dans le contexte des années quatre-vingt, il s'agissait d'abord pour les organisations d'instaurer une communication interne qui rende l'organisation plus performante: celle-ci prise dans une double contrainte -intensification de la concurrence et hausse des coûts de production (en France et dans plusieurs pays avancés) - comptait pour sa survie sur une meilleure productivité du facteur travail. Les acteurs mieux informés de la logique des prises de décision en interne, des relations avec l'externe, et de la politique globale, pouvaient replacer l'intérêt de leur tâche dans un contexte, participer à l'amélioration des processus, suggérer des solutions nouvelles, avoir une réponse plus rapide et plus pertinente aux sollicitations extérieures, assurer une qualité de production et de service sinon totale du moins maximale. 
5 Les années quatre-vingt dix confrontent ces objectifs ambitieux à la réalité des faits. Le cortège de faillites d'entreprise et la hausse inexorable du chômage posent problème. À qui revient la faute? Les causes sont nombreuses et la prudence dans la réponse est de rigueur. Les acteurs interpellent les universitaires pour qu'ils éclairent leur réflexion : la communication ne semble pas avoir joué le rôle qu'on attendait d'elle. L'outil était-il mal conçu? Mal utilisée L'audit préalable était-il pratiqué avec assez de soin? La communication s'intégrait-elle suffisamment aux autres pratiques managériales? À moins que ce ne fût l'analyse du contexte qui fût défaillante? Ou que la démarche fût décridibilisée par une éthique insuffisante ${ }^{5}$ ?

6 Les auteurs des articles élaborent des analyses pour répondre à ces questions ; ils savent qu'elles sont d'autant plus attendues que faute d'explication, les investissements lourds ${ }^{6}$ en communication et certains recrutements subissent un temps d'arrêt. Pourtant, par rigueur intellectuelle, ils souhaitent s'abstraire du court terme.

7 En premier lieu il leur semble indispensable :

8 - de retravailler la définition de la « communication interne » en la modulant selon les organisations et les approches de la communication (N. Giroux),

9 - de souligner qu'il existe une « littérature » sur le sujet (et plus généralement sur la communication d'entreprise) qui, inspirée de la pratique des entreprises, les influence à partir de jeux de territoires et d'enjeux (Y. Nicolas),

10 - d'évaluer les pratiques d'audit (G. Willet) dont on sait qu'elles fondent en principe le diagnostic sur lequel repose la politique de communication, évaluation qui pose problème si on observe (avec V. Carayol) qu'elle devrait impliquer que l'on puisse s'appuyer sur une stratégie formalisée et que celle-ci n'est pas toujours énoncée.

11 Ils se demandent ensuite si l'échec-relatif-de la communication ne tient pas aussi au fait qu'il s'agissait moins de résoudre des problèmes conjoncturels que d'accompagner des évolutions structurelles; ils analysent au passage :

12 - le lien entre communication interne et évolution de la gestion des ressources humaines (A. Labruffe, A. Lafrance, E.M. Hernandez),

13 - la révolution télématique qui remet en question les méthodes de travail et la conception de l'organisation (F. Jauréguiberry),

14 - la télécommunication qui nous réunit tous autour des mêmes signaux et nous conduit à homogénéiser le langage,

15 - l'uniformisation des consommations et des cultures des biens qui, n'ayant plus de frontières, circulent au meilleur rapport coût/qualité/prix, transforment le monde en 'village planétaire » et déplacent les effets d'échelle de la communication.

16 La communication ne constitue pas seulement un investissement en produits de communication; c'est aussi un investissement immatériel ${ }^{7}$ auquel l'organisation ne consent que si elle en voit les effets-retour en termes financiers. Tandis que la France marque un temps d'arrêt et s'interroge les masses financières à lui consacrer, les Japonais ${ }^{8}$ continuent dans le tout communication: ils proposent le terme de Humanware ${ }^{9}$ pour cerner la part de productivité que l'entreprise japonaise dégage à partir de ce type d'investissement ${ }^{10}$.

$17 \mathrm{Au}$ nom de la compétitivité industrielle du Japon faut-il à nouveau nous inspirer des nouvelles formes de management qu'il propose? 


\section{NOTES}

1. Les noms des auteurs des articles du dossier sont présentés dans le corps du texte, ceux des autres rubriques en note

2. CNE Les Sciences de l'information et de la Communication, 1993, Paris

3. Sciences de l'Information et de la Communication

4. Cf articles de Ch Michon, E-M Hernandez, R de Mari-court

5. Cf l'article de P. Lamarque: Entre fonction et profession: Pour une nouvelle pensée de la communication des organisations, dans la rubrique Analyses et in Les désordres du sens, alerte sur les médias, les entreprises, la vie publique ", 1993, Paris, ESF.

6. La mise en place de réseaux interne ou la réalisation de films d'entreprise rentrent dans ce cadre.

7. Le conseil national de l'information statistique définit » un investissement immatériel comme une dépense qui, bien qu'inscrite en charge d'exploitation, développe la capacité de production et valorise l'entreprise en s'accumulant sous la forme d'un capital ammortissable sur une production future et en constituant une valeur patrimoniable cessible sur le marché ».Rapport sur les comptes de la Nation, INSEE, 1993

8. Cf l'article de R. de Maricourt Communication interne et culture d'entreprise au Japon in rubrique Analyses

9. «Système productif : nouveaux principes et diversités nationales »,Extrait du rapport de l'OCDE » La technologie et l'économie. Les relations déterminantes », publié par Problèmes économiques, mars 1994, p55-60.

10. ou de tout investissement similaire provoquant l'amélioration de l'interaction entre les ressources humaines de l'entreprise.

11. et des représentations dans les ouvrages de vulgarisation (cf l'article de H. Hotier dans Analyses, et l'étude par les étudiants de la section communication interne du DESS de l'ISIC, des enquêtes publiées en juin 1993 par Entreprise et Carrières et les analyses bibliographiques des dernières pages de la revue).

12. Cf aussi dans Etat de la recherche, la synthèse des travaux du groupe «Communication et réflexion » 